\title{
Grenzgänge zwischen Beruf und Privatleben
}

Im Kinofilm Finsteres Glück nimmt die klinische Psychologin Eliane Hess ihren achtjährigen Patienten Yves, der gerade seine ganze Familie verloren hat, privat bei sich auf. Die Grenzen ihres beruflichen Auftrags, dem traumatisierten Kind bei der Verarbeitung beizustehen, verschwimmen mit ihren privaten Wünschen und ihrer Zuneigung zum kleinen Jungen. Ausgehend von diesem Zentraldilemma der Geschichte machen sich Iris Ritzmann und Eberhard Wolff hier - je für sich - ihre Gedanken über die Grenze zwischen therapeutischem Beruf und Privatleben sowie deren Überschreitung.

\section{Berufliches Engagement nach Feierabend}

Wo liegen die Grenzen zwischen professioneller Distanz und persönlicher Betroffenheit? «Wenn die Sorgen Ihrer Patienten Sie auch nach Feierabend noch beschäftigen, haben Sie zum Beruf zu wenig Distanz.» Der Berufsberater, durch einen unfreiwilligen Jobverlust in mein Leben getreten, drückte sich deutlich aus. Die Patientinnen, denen ich seither stundenweise zur Seite stehe, begleiten mich gedanklich allerdings auch in meiner Freizeit. Bin ich als Ärztin deshalb unprofessionell? Gerne tausche ich mich mit befreundeten Berufskolleginnen und -kollegen über ihre Praxistätigkeit aus. In unseren Erzählungen geht es um Menschen, die uns berühren, denen wir helfen möchten und die uns zum Nachdenken anregen. Und genauso sprechen wir über Schwierigkeiten von Freunden und Angehörigen. Wir engagieren uns, nehmen emotional Anteil und suchen nach Lösungswegen, in der Regel auch nach Feierabend.

Wie professionell verhält sich die Therapeutin im Film? Sie kann die Konsequenzen ihres Handelns schwer abschätzen und gerät zusehends ins Taumeln. Hat ihre instabile psychische Lebenslage überhaupt etwas mit ihrer Beziehung zum kleinen Jungen zu tun? Oder ist es sogar umgekehrt, dass sie von dem Kind Zuneigung und Bewunderung erwartet? Fast scheint es, als ob der kleine Patient seiner Therapeutin den Sinn ihres Lebens aufzeigen sollte. Vielleicht liegt Professionalität vor allem darin, bewusst mit eigenen Gefühlen umzugehen und auf die Bedürfnisse eines hilfesuchenden Menschen einzutreten, ohne selbst den Boden unter den Füssen zu verlieren.

\section{Therapie zwischen Dienst und Schnaps}

Mein Vater, er wäre jetzt hundert, war der Inbegriff eines deutschen Steuerbeamten. «Dienst ist Dienst, und Schnaps ist Schnaps», hörte ich ihn früher immer wieder sagen. Doch zum Mittagessen im Familienkreis genehmigte er sich regelmässig ein oder zwei Gläschen «Dienst» mit Erzählungen von der Arbeit. Ein ThomasMann-Zitat verhiess ihm den Ausweg aus dem Dilemma: «Nur wer die Form beherrscht, darf sich darüber hinwegsetzen.»

Gerade in den Berufen, die eng mit Menschen umgehen, weiss man, dass Dienst und Schnaps getrennt werden sollen, um professionellen Desastern zu entgehen. Aber spätestens heute weiss man dort genauso, dass dies ein frommer Wunsch ist. In einem hoffentlich engagierten Berufsleben fliessen Dienst und Schnaps irgendwie auch ineinander. Es kommt darauf an, die Vermischungsbereiche souverän zu beherrschen. Ich hoffe übrigens, dass ich diesem Anspruch hier selber gerecht werde.

Die Therapeutin im Film Finsteres Glück bietet zumindest an, den Jungen als «Fall» abzugeben. Doch wo der finstere Film glücklich endet, fängt die spannende Geschichte für uns eigentlich erst an. Was passiert nach der «letzten Klappe»? Was erhalten die nachfolgenden kleinen Patienten der Eliane Hess, die ihr auch ans Herz gehen? Wie wird sie ihre persönliche Zuneigung auf unterschiedliche Patienten verteilen? Und zu Hause? Erhält Yves ein paar Gläschen Psychotherapie am Mittagstisch? Wo legt sie die Grenze zwischen Dienst und Schnaps, damit Yves nicht eines Tages seiner Pflegemutter in die Augen schaut und denkt: Das war der Beginn einer langen Krise.

Eberhard Wolff 